\title{
Approximation properties of multivariate exponential sampling series
}

\author{
Kurşun S. ${ }^{1}$, Turgay M. ${ }^{1}$, Alagöz O. ${ }^{2}$, Acar T. ${ }^{1, 凶}$
}

\begin{abstract}
In this paper, we generalize the family of exponential sampling series for functions of $n$ variables and study their pointwise and uniform convergence as well as the rate of convergence for the functions belonging to space of log-uniformly continuous functions. Furthermore, we state and prove the generalized Mellin-Taylor's expansion of multivariate functions. Using this expansion we establish pointwise asymptotic behaviour of the series by means of Voronovskaja type theorem.

Key words and phrases: multivariate exponential sampling series, rate of convergence, multivariate Mellin-Taylor formula, Voronovskaja type theorem.
\end{abstract}

\footnotetext{
${ }^{1}$ Selçuk University, 42130 Selçuklu-Konya, Turkey

2 Bilecik Şeyh Edebali University, 11230 Bilecik, Turkey

$\checkmark$ Corresponding author

E-mail: sadettinkursun@yahoo.com(Kurşun S.), metinturgay@yahoo.com (Turgay M.), osman.alagoz@bilecik.edu.tr(Alagöz O.), tunceracar@ymail.com(Acar T.)
}

\section{Introduction}

The sampling series and their applications are the most challenging theory in approximation theory and especially in signal and image processing. The classical sampling theorem had been intensively studied by P.L. Butzer and his research group though generally attributed to E.T. Whittaker, V.A. Kotel'nikov and C.E. Shannon. The most common method was given by P.L. Butzer (see [18]) named generalized sampling series as

$$
\left(S_{w}^{\chi} f\right)(t):=\sum_{k \in \mathbb{Z}} f\left(\frac{k}{w}\right) \chi(w t-k), \quad t \in \mathbb{R}, \quad w>0,
$$

where the function $\chi$ is called kernel function and satisfies some assumptions of approximate identities. The generalized sampling series became most prominent working area in a very short time thanks to its wide applications. We refer the readers to $[7,15,19,20]$ and references therein for some applications and forms of generalized sampling series.

In order to expand applications areas, P.L. Butzer et al. introduced generalized sampling series for multivariate signals in [15]. The multivariate generalized sampling series are defined by

$$
\left(S_{\mathbf{w}}^{\varphi} f\right)(\mathbf{t}):=\frac{1}{(\sqrt{2 \pi})^{n}} \sum_{\mathbf{k} \in \mathbb{Z}^{n}} f\left(\frac{\mathbf{k}}{\mathbf{w}}\right) \varphi(\mathbf{w} \mathbf{t}-\mathbf{k}), \quad \mathbf{t} \in \mathbb{R}^{n}, \mathbf{w} \in \mathbb{R}_{+}^{n},
$$

such that $\varphi: \mathbb{R}^{n} \rightarrow \mathbb{C}$ is a continuous and bounded kernel function and $f$ is a continuous function. For some distinct treatment of multivariate generalized sampling series to applications, the readers can see also $[13,16,24]$. 
The pioneer studies of exponential sampling theorem in the theory of Mellin transform were given in [23] and further considerations were deeply given in [17]. To overcome Mellin band-limited restrictiveness of $f($ see $[3,4]), C$. Bardaro et al. [8] established a new generalization of exponential sampling theorem by replacing $\operatorname{lin}_{c}$ with an arbitrary function $\varphi$ satisfying suitable assumptions. In [14, 21], a series representations of Mellin band-limited functions were established via their exponentially spaced samples, which was considered as Mellin version of the Shannon sampling theorem. Among the others, we can refer the readers to [5, 6, 8, 12].

Furthermore, C. Bardaro et al. [2] introduced generalized exponential sampling series for bivariate functions and obtained some convergence results. They also proved Mellin-Taylor formula for bivariate functions and applied it to the pointwise asymptotic behaviour of the series. As an application, the magnitude of an earthquake through the behaviour of the seismic waves was established by bivariate generalized exponential sampling series.

The treatment of the theory in multivariate setting is important also from the point of view of the applications; indeed in signal theory, in order to deal with image processing, one has to work with multivariate signals. In this paper, firstly we introduce multidimensional exponential sampling series and study their pointwise, uniform convergence and the rate of convergence by expanded logarithmic modulus of continuity of target function. Secondly, since exponential sampling theorem for Mellin band-limited functions represents a Mellin version of the classical Shannon sampling theorem of Fourier analysis [25, 22], we present multidimensional Mellin-Taylor formula with its proof and obtain Voronovskaja type theorem for new operators by using the formula.

\section{Construction of operators}

Let us denote by $\mathbb{N}^{n}, \mathbb{N}_{0}^{n}$ and $\mathbb{Z}^{n}$ the sets of vectors $\mathbf{k}=\left(k_{1}, k_{2}, \ldots, k_{n}\right)$ with $k_{i}, i=1,2, \ldots, n$, positive integers, nonnegative integers and integers, respectively and we set $\lfloor k\rfloor:=\sum_{k=1}^{n} k_{i}$. Moreover, by $\mathbb{R}^{n}$ we will denote the $n$ dimensional Euclidean space consisting of all vectors $\left(x_{1}, x_{2}, \ldots, x_{n}\right)$ with $x_{i} \in \mathbb{R}$ for $i=1,2, \ldots, n$.

Let $\mathbf{x}=\left(x_{1}, x_{2}, \ldots, x_{n}\right), \mathbf{y}=\left(y_{1}, y_{2}, \ldots, y_{n}\right) \in \mathbb{R}^{n}$, then we say that $\mathbf{x}>\mathbf{y}$ if and only if $x_{i}>y_{i}$ for $i=1,2, \ldots, n$ and we will denote by $\mathbf{1}:=(1,1, \ldots, 1), \mathbf{0}:=(0,0, \ldots, 0)$ and by $\mathbb{R}_{+}^{n}$ the space of all vectors $\mathbf{x}>\mathbf{0}$. Given $\mathbf{x}, \mathbf{y} \in \mathbb{R}^{n}$ and $\alpha \in \mathbb{R}$ the usual operations are given by

$$
\mathbf{x}+\mathbf{y}:=\left(x_{1}+y_{1}, x_{2}+y_{2}, \ldots, x_{n}+y_{n}\right), \quad \alpha \mathbf{x}:=\left(\alpha x_{1}, \alpha x_{2}, \ldots, \alpha x_{n}\right) .
$$

Further, the product and division of two vectors of $\mathbb{R}^{n}$ defined as

$$
\mathbf{x y}:=\left(x_{1} y_{1}, x_{2} y_{2}, \ldots, x_{n} y_{n}\right), \quad \frac{\mathbf{x}}{\mathbf{y}}:=\left(\frac{x_{1}}{y_{1}}, \frac{x_{2}}{y_{2}}, \ldots, \frac{x_{n}}{y_{n}}\right), \quad y_{i} \neq 0 \text { for all } i=1,2, \ldots, n \text {. }
$$

Let $\alpha^{\mathbf{x}}:=\left(\alpha^{x_{1}}, \alpha^{x_{2}}, \ldots, \alpha^{x_{n}}\right)$ with $\alpha>0, \mathbf{x}^{\mathbf{y}}:=\left(x_{1}^{y_{1}}, \ldots, x_{n}^{y_{n}}\right)$ and

$$
\log (\mathbf{x}):=\left(\log \left(x_{1}\right), \log \left(x_{2}\right), \ldots, \log \left(x_{n}\right)\right) \quad \text { with } \quad \mathbf{x}>\mathbf{0} .
$$

The norm of a vector $\mathbf{x} \in \mathbb{R}^{n}$ is given by $\|\mathbf{x}\|:=\sqrt{x_{1}^{2}+x_{2}^{2}+\ldots+x_{n}^{2}}$, and the Euclidean distance is defined by $d(\mathbf{x}, \mathbf{y}):=\|\mathbf{x}-\mathbf{y}\|$. For $\mathbf{w}=\left(w_{1}, w_{2}, \ldots, w_{n}\right) \in \mathbb{R}_{+}^{n}$, by the notion of $\mathbf{w} \rightarrow+\infty$, we mean that $\bar{w}:=\min \left\{w_{1}, w_{2}, \ldots, w_{n}\right\} \rightarrow+\infty$.

Let $I$ be an interval of $\mathbb{R}^{n}$ (bounded or not), we denote by $C(I)$ the space of all continuous and bounded functions on $I$. Moreover, for $m \in \mathbb{N}$ we denote $C^{(m)}(I)$ as the subspace of $C(I)$ which consists of all functions $f$ with the continuous derivatives up to the order $m$. 
A function $f: I \rightarrow \mathbb{C}$ is called log-uniformly continuous function on $I$, if for every $\varepsilon>0$ there exists $\delta(\varepsilon)>0$ such that $|f(\mathbf{x})-f(\mathbf{y})|<\varepsilon$ whenever $\|\log (\mathbf{x})-\log (\mathbf{y})\| \leq \delta(\varepsilon)$, $\mathbf{x}, \mathbf{y} \in \mathbb{R}_{+}^{n}$. By $\mathcal{C}(I)$, we denote the space of all log-uniformly continuous and bounded functions on $I$. It is obvious that the notion of log-uniform continuity is equivalent to the classical uniform continuity on compact intervals $I \subset \mathbb{R}_{+}^{n}$ (see $[23,11]$ ).

Let $\varphi: \mathbb{R}_{+}^{n} \rightarrow \mathbb{R}$ be a continuous function satisfying the following assumptions:

$$
\sum_{\mathbf{k} \in \mathbb{Z}^{n}} \varphi\left(e^{-\mathbf{k}} \mathbf{x}\right)=\sum_{\left(k_{1}, k_{2}, \ldots, k_{n}\right) \in \mathbb{Z}^{n}} \varphi\left(e^{-k_{1}} x_{1}, e^{-k_{2}} x_{2}, \ldots, e^{-k_{n}} x_{n}\right)=1
$$

for every $\mathbf{x}=\left(x_{1}, x_{2}, \ldots, x_{n}\right) \in \mathbb{R}_{+}^{n} ;$

( $\varphi .2)$ there holds

$$
M_{0}(\varphi):=\sup _{\mathbf{x} \in \mathbb{R}_{+}^{n}} \sum_{\mathbf{k} \in \mathbb{Z}^{n}}\left|\varphi\left(e^{-\mathbf{k}} \mathbf{x}\right)\right|<+\infty
$$

uniformly with respect to $\mathbf{x}$.

$$
\lim _{r \rightarrow+\infty} \sum_{\|\mathbf{k}-\log (\mathbf{x})\|>r}\left|\varphi\left(e^{-\mathbf{k}} \mathbf{x}\right)\right|=0
$$

Let $\Phi$ denotes the class of all kernel functions $\varphi$ satisfying the assumptions $(\varphi \cdot 1)-(\varphi .3)$. Then, multidimensional exponential sampling series can be defined for $\varphi \in \Phi$ by

$$
\left(\mathrm{E}_{\mathbf{w}}^{\varphi} f\right)(\mathbf{x}):=\sum_{\mathbf{k} \in \mathbb{Z}^{n}} f\left(e^{\frac{\mathbf{k}}{\mathbf{w}}}\right) \varphi\left(e^{-\mathbf{k}} \mathbf{x}^{\mathbf{w}}\right)
$$

for any $\mathbf{x}=\left(x_{1}, x_{2}, \ldots, x_{n}\right) \in \mathbb{R}_{+}^{n}, \mathbf{w}=\left(w_{1}, w_{2}, \ldots, w_{n}\right) \in \mathbb{R}_{+}^{n}$ and $f: \mathbb{R}_{+}^{n} \rightarrow \mathbb{C}$, which can be equivalently stated as

$$
\left(\mathrm{E}_{\mathbf{w}}^{\varphi} f\right)(\mathbf{x}):=\sum_{\left(k_{1}, k_{2}, \ldots, k_{n}\right) \in \mathbb{Z}^{n}} f\left(e^{\frac{k_{1}}{w_{1}}}, e^{\frac{k_{2}}{w_{2}}}, \ldots, e^{\frac{k_{n}}{w_{n}}}\right) \varphi\left(e^{-k_{1}} x_{1}^{w_{1}}, e^{-k_{2}} x_{2}^{w_{2}}, \ldots, e^{-k_{n}} x_{n}^{w_{n}}\right) .
$$

Remark 1. If $f$ is bounded, that is $|f(\mathbf{x})| \leq N$ for every $\mathbf{x} \in \mathbb{R}_{+}^{n}$, then the operator (1) is well-defined.

It is easily proved by the hypothesis and the assumption of ( $\varphi .2)$. Indeed,

$$
\begin{aligned}
\left|\left(\mathrm{E}_{\mathbf{w}}^{\varphi} f\right)(\mathbf{x})\right| & \leq \sum_{\left(k_{1}, k_{2}, \ldots, k_{n}\right) \in \mathbb{Z}^{n}}\left|f\left(e^{\frac{k_{1}}{w_{1}}}, e^{\frac{k_{2}}{w_{2}}}, \ldots, e^{\frac{k_{n}}{w_{n}}}\right)\right|\left|\varphi\left(e^{-k_{1}} x_{1}^{w_{1}}, e^{-k_{2}} x_{2}^{w_{2}}, \ldots, e^{-k_{n}} x_{n}^{w_{n}}\right)\right| \\
& \leq N \sum_{\left(k_{1}, k_{2}, \ldots, k_{n}\right) \in \mathbb{Z}^{n}}\left|\varphi\left(e^{-k_{1}} x_{1}^{w_{1}}, e^{-k_{2}} x_{2}^{w_{2}}, \ldots, e^{-k_{n}} x_{n}^{w_{n}}\right)\right|<+\infty .
\end{aligned}
$$

Remark 2. Considering the definition of the operators (1), we immediately have that

$$
\left(\mathrm{E}_{\mathbf{w}}^{\varphi} \tilde{\mathbf{1}}\right)(\mathbf{x})=1,
$$

where $\tilde{\mathbf{1}}(\mathbf{x}):=1$ for every $\mathbf{x} \in \mathbb{R}_{+}^{n}$.

Here, we note that a Kantorovich form of the operators (1) has been recently introduced and studied in [1]. 


\section{Pointwise and uniform convergence of $\left(E_{w}^{\varphi}\right)$}

In this section, we present two main theorems about pointwise and uniform convergence for the family of multivariate exponential sampling operators $\left(E_{\mathbf{w}}^{\varphi}\right), \mathbf{w}>\mathbf{0}$.

Theorem 1. Let $f$ be a bounded function. Then

$$
\lim _{\mathbf{w} \rightarrow+\infty}\left(E_{\mathbf{w}}^{\varphi} f\right)(\mathbf{x})=f(\mathbf{x})
$$

holds at each continuity point $\mathbf{x} \in \mathbb{R}_{+}^{n}$ of $f$.

Proof. Let $\varepsilon>0$ be fixed. Since $f \in C\left(\mathbb{R}_{+}^{n}\right)$, by the continuity of $f$ at $\mathbf{x}$ there exists $\delta=\delta(\varepsilon)$ such that $\left|f\left(e^{\frac{\mathbf{k}}{\mathbf{w}}}\right)-f(\mathbf{x})\right|<\varepsilon$ whenever $\left\|\log \left(e^{\frac{\mathbf{k}}{\mathbf{w}}}\right)-\log (\mathbf{x})\right\|=\left\|\frac{\mathbf{k}}{\mathbf{w}}-\log (\mathbf{x})\right\| \leq \delta$. Вy $(\varphi \cdot 1)$, we can write

$$
\begin{aligned}
\left|\left(\mathrm{E}_{\mathbf{w}}^{\varphi} f\right)(\mathbf{x})-f(\mathbf{x})\right| & \leq \sum_{\mathbf{k} \in \mathbb{Z}^{n}}\left|f\left(e^{\frac{\mathbf{k}}{\mathbf{w}}}\right)-f(\mathbf{x})\right|\left|\varphi\left(e^{-\mathbf{k} \mathbf{x}^{\mathbf{w}}}\right)\right| \\
& =\left\{\sum_{\left\|\frac{\mathbf{k}}{\mathbf{w}}-\log (\mathbf{x})\right\| \leq \delta}+\sum_{\left\|\frac{\mathbf{k}}{\mathbf{w}}-\log (\mathbf{x})\right\|>\delta}\right\}\left|f\left(e^{\frac{\mathbf{k}}{\mathbf{w}}}\right)-f(\mathbf{x})\right|\left|\varphi\left(e^{-\mathbf{k}} \mathbf{x}^{\mathbf{w}}\right)\right|=: I_{1}+I_{2} .
\end{aligned}
$$

By using the assumption of $(\varphi .2)$, we have $I_{1}<M_{0}(\varphi) \mathcal{E}$. Now let us estimate $I_{2}$. By taking into account that

$$
\left\|\frac{\mathbf{k}}{\mathbf{w}}-\log (\mathbf{x})\right\| \leq \frac{\|\mathbf{k}-\mathbf{w} \log (\mathbf{x})\|}{\bar{w}}
$$

and the assumption $(\varphi .3)$, for sufficiently large $\bar{w}>0$ we have

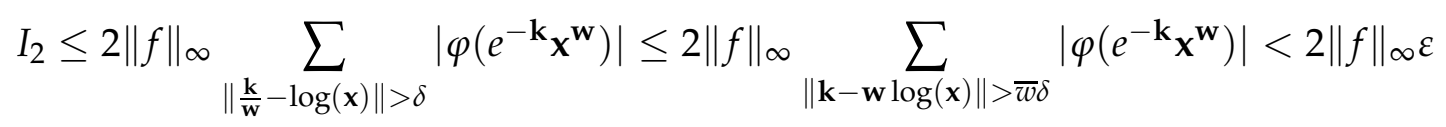

and this completes the proof.

Now, we give a uniform convergence result by the following theorem in case of $f$ belongs to log-uniformly continuous and bounded functions space.

Theorem 2. Let $f \in \mathcal{C}\left(\mathbb{R}_{+}^{n}\right)$ and $\varphi \in \Phi$, then

$$
\lim _{\mathbf{w} \rightarrow+\infty}\left\|\left(\mathrm{E}_{\mathbf{w}}^{\varphi} f\right)-f\right\|_{\infty}=0
$$

Proof. We omit the proof details because it is similar to the proof of Theorem 1.

\section{Rate of convergence of $\left(\mathrm{E}_{\mathrm{w}}^{\varphi}\right)$}

This section is devoted to determine a rate of convergence of the family $\left(\mathrm{E}_{\mathbf{w}}^{\varphi}\right)$. Firstly, we recall and define required tools. The logarithmic modulus of continuity of $f \in \mathcal{C}\left(\mathbb{R}_{+}\right)$is defined in [10]. Here, we generalize the definition of logarithmic modulus of continuity for the functions $f \in \mathcal{C}\left(\mathbb{R}_{+}^{n}\right)$. For $\delta>0$ we define

$$
\omega(f, \delta):=\sup \left\{|f(\mathbf{x})-f(\mathbf{y})|: \mathbf{x}, \mathbf{y} \in \mathbb{R}_{+}^{n},\|\log (\mathbf{x})-\log (\mathbf{y})\| \leq \delta\right\} .
$$

We give some properties of logarithmic modulus of the continuity for multivariate functions by the following lemma. The proof of the lemma can be easily obtained by using the similar concept for single valued functions (see [8]). 
Lemma 1. For a function $f \in \mathcal{C}\left(\mathbb{R}_{+}^{n}\right)$, logarithmic modulus of continuity has the following properties:

(i) for each $\delta>0, \omega(f, \delta)$ is finite;

(ii) if $\delta_{1} \leq \delta_{2}$, then $\omega\left(f, \delta_{1}\right) \leq \omega\left(f, \delta_{2}\right)$;

(iii) for $\delta_{1}>0$ and $\delta_{2}>0, \omega\left(f, \delta_{1}+\delta_{2}\right) \leq \omega\left(f, \delta_{1}\right)+\omega\left(f, \delta_{2}\right)$ holds;

(iv) for every $m \in \mathbb{N}, \omega(f, m \delta) \leq m \omega(f, \delta)$ holds;

(v) for every $\lambda>0, \omega(f, \lambda \delta) \leq(1+\lambda) \omega(f, \delta)$;

(vi) for any $\mathbf{t}, \mathbf{x} \in \mathbb{R}_{+}^{n},|f(\mathbf{t})-f(\mathbf{x})| \leq \omega(f, \| \log (\mathbf{t})-\log (\mathbf{x})||)$;

(vii) for any $\mathbf{x}, \mathbf{y} \in \mathbb{R}_{+}^{n},|f(\mathbf{x})-f(\mathbf{y})| \leq(1+\|\log (\mathbf{x})-\log (\mathbf{y})\| / \delta) \omega(f, \delta)$;

(viii) $\lim _{\delta \rightarrow 0} \omega(f, \delta)=0$.

Theorem 3. If $\varphi$ is a kernel function satisfying

$$
M_{1}(\varphi):=\sup _{\mathbf{x} \in \mathbb{R}_{+}^{n}} \sum_{\mathbf{k} \in Z^{n}}\left|\varphi\left(e^{-\mathbf{k}} \mathbf{x}\right)\right|\|\mathbf{k}-\log (\mathbf{x})\|<+\infty
$$

and if $f \in \mathcal{C}\left(\mathbb{R}_{+}^{n}\right)$, then

$$
\left|\left(\mathrm{E}_{\mathbf{w}}^{\varphi} f\right)(\mathbf{x})-f(\mathbf{x})\right| \leq \omega\left(f, \frac{1}{\bar{w}}\right)\left(M_{0}(\varphi)+M_{1}(\varphi)\right)
$$

holds for every $\bar{w}>0$.

Proof. By the definition of the family of operators (1), the fact $\bar{w} \leq\|\mathbf{w}\|$ and hypothesis (2), we have that

$$
\begin{aligned}
\left|\left(\mathrm{E}_{\mathbf{w}}^{\varphi} f\right)(\mathbf{x})-f(\mathbf{x})\right| & \leq \sum_{\mathbf{k} \in \mathbb{Z}^{n}}\left|f\left(e^{\frac{\mathbf{k}}{\mathbf{w}}}\right)-f(\mathbf{x})\right|\left|\varphi\left(e^{-\mathbf{k}} \mathbf{x}^{\mathbf{w}}\right)\right| \leq \sum_{\mathbf{k} \in \mathbb{Z}^{n}} \omega\left(f,\left\|\frac{\mathbf{k}}{\mathbf{w}}-\log (\mathbf{x})\right\|\right)\left|\varphi\left(e^{-\mathbf{k}} \mathbf{x}^{\mathbf{w}}\right)\right| \\
& \leq \omega\left(f, \frac{1}{\bar{w}}\right) \sum_{\mathbf{k} \in \mathbb{Z}^{n}}\left(1+\bar{w} \frac{\|\mathbf{k}-\mathbf{w} \log (\mathbf{x})\|}{\|\mathbf{w}\|}\right)\left|\varphi\left(e^{-\mathbf{k}} \mathbf{x}^{\mathbf{w}}\right)\right| \\
& \leq \omega\left(f, \frac{1}{\bar{w}}\right)\left(M_{0}(\varphi)+M_{1}(\varphi)\right) .
\end{aligned}
$$

\section{Multidimensional Mellin-Taylor formula and Voronovskaja type theo- rem}

In this section, we will use the following notations.

For a given $\mathbf{x} \in \mathbb{R}_{+}^{n}$ and $\mathbf{h}=\left(h_{1}, \ldots, h_{n}\right) \in \mathbb{N}_{0}^{n}$ we denote $\langle\mathbf{x}\rangle:=\prod_{i=1}^{n} x_{i}$ and $\mathbf{h} !=h_{1} ! \ldots h_{n} !$

Let $f: \mathbb{R}_{+}^{n} \rightarrow \mathbb{C}$. The first order of Mellin derivative of $f$ with respect to the variable $x_{i}, i=1, \ldots, n$ at the point $\mathbf{x}=\left(x_{1}, \ldots, x_{n}\right)$ is given by

$$
\Theta_{x_{i}} f(\mathbf{x}):=x_{i} \frac{\partial f(\mathbf{x})}{\partial x_{i}}
$$


(see [2]). For a given $\mathbf{k}=\left(k_{1}, \ldots, k_{n}\right) \in \mathbb{N}_{0}^{n}$, we define the partial Mellin derivatives of order $r=k_{1}+\ldots+k_{n}$ at the point $\mathbf{x}$ as

$$
\Theta_{x_{1}^{k_{1} \ldots x_{n}}}^{k_{n}} f(\mathbf{x}):=\Theta_{x_{1}}^{k_{1}}\left(\Theta_{x_{2}}^{k_{2}} \cdots\left(\Theta_{x_{n}}^{k_{n}} f\right)\right)(\mathbf{x})
$$

and we will put $\Theta_{x_{i}}^{1} f(\mathbf{x}):=\Theta_{x_{i}} f(\mathbf{x}), \Theta_{x_{i}}^{0} f(\mathbf{x}):=f(\mathbf{x})$.

In order to construct multidimensional Mellin-Taylor formula, which introduced in [9] for an one-dimensional space, we give the following notation. For a given $\mathbf{x}=\left(x_{1}, \ldots, x_{n}\right)$ and $\mathbf{t}=\left(t_{1}, \ldots, t_{n}\right)$ we set

$$
\begin{aligned}
& \left(\Theta_{x_{1}} \log \left(t_{1}\right)+\ldots+\Theta_{x_{n}} \log \left(t_{n}\right)\right)^{m} f(\mathbf{x}) \\
& \quad:=\sum_{\lfloor\mathbf{h}\rfloor=m} \frac{m !}{\mathbf{h} !} \Theta_{x_{1}}^{h_{1}}\left(\ldots\left(\Theta_{x_{n}}^{h_{n}} f\right)\right)(\mathbf{x}) \log ^{h_{1}}\left(t_{1}\right) \ldots \log ^{h_{n}}\left(t_{n}\right)
\end{aligned}
$$

with $m \in \mathbb{N}$ and $f \in C^{(m)}\left(\mathbb{R}_{+}^{n}\right)$ locally at $\mathbf{x}=\left(x_{1}, \ldots, x_{n}\right)$.

Proposition 1. Let $m \in \mathbb{N}$ and $f: \mathbb{R}_{+}^{n} \rightarrow \mathbb{C}$ be a function in $C^{(m)}\left(\mathbb{R}_{+}^{n}\right)$. Then for $\mathbf{x}=\left(x_{1}, \ldots, x_{n}\right) \in \mathbb{R}_{+}^{n}$ and $\mathbf{t}=\left(t_{1}, \ldots, t_{n}\right) \in \mathbb{R}_{+}^{n}$, we have

$$
f(\mathbf{t x})=f(\mathbf{x})+\sum_{r=1}^{m-1} \frac{1}{r !}\left(\Theta_{x_{1}} \log \left(t_{1}\right)+\ldots+\Theta_{x_{n}} \log \left(t_{n}\right)\right)^{r} f(\mathbf{x})+R_{m}(\mathbf{t})
$$

with Lagrange remainder

$$
R_{m}(\mathbf{t})=\frac{1}{m !}\left(\Theta_{x_{1}} \log \left(t_{1}\right)+\ldots+\Theta_{x_{n}} \log \left(t_{n}\right)\right)^{m} f\left(\xi_{1}, \ldots, \xi_{n}\right),
$$

where $\left(\xi_{1}, \ldots, \xi_{n}\right)$ is a suitable point in the segment $L_{t_{1} \ldots t_{n}}$ with the endpoints $\left(x_{1}, \ldots, x_{n}\right)$, $\left(t_{1} x_{1}, \ldots, t_{n} x_{n}\right)$.

Proof. Without loss of generality we prove the proposition for $m=2$. Let us consider the function $F(z)=f\left(t_{1}^{\log (z)} x_{1}, t_{2}^{\log (z)} x_{2}, \ldots, t_{n}^{\log (z)} x_{n}\right)$ with $z \in[1, e]$. By applying one dimensional Mellin-Taylor formula with Lagrange remainder, we get

$$
F(z)=F(1)+\Theta F(1) \log (z)+\frac{\Theta^{2} F(\tilde{z})}{2} \log ^{2}(z)
$$

with $\tilde{z} \in(1, e)$. By using Mellin derivative, we obtain

For $z=1$, we get

$$
\begin{aligned}
\Theta F(z)= & \frac{\partial f}{\partial x_{1}}\left(t_{1}^{\log (z)} x_{1}, \ldots, t_{n}^{\log (z)} x_{n}\right) x_{1} t_{1}^{\log (z)} \log \left(t_{1}\right) \\
& +\ldots+\frac{\partial f}{\partial x_{n}}\left(t_{1}^{\log (z)} x_{1}, \ldots, t_{n}^{\log (z)} x_{n}\right) x_{n} t_{n}^{\log (z)} \log \left(t_{n}\right) .
\end{aligned}
$$

$$
\begin{aligned}
\Theta F(1)= & \Theta_{x_{1}} f(\mathbf{x}) \log \left(t_{1}\right)+\ldots+\Theta_{x_{n}} f(\mathbf{x}) \log \left(t_{n}\right) \\
\Theta^{2} F(z)= & \sum_{i=1}^{n} \frac{\partial^{2} f}{\partial x_{i}^{2}}\left(t_{1}^{\log (z)} x_{1}, \ldots, t_{n}^{\log (z)} x_{n}\right) x_{i}^{2} t_{i}^{2 \log (z)} \log ^{2}\left(t_{i}\right) \\
& +\sum_{i, j=1, i \neq j}^{n} \frac{\partial^{2} f}{\partial x_{i} \partial x_{j}}\left(t_{1}^{\log (z)} x_{1}, \ldots, t_{n}^{\log (z)} x_{n}\right) x_{i} x_{j}\left(t_{i} t_{j}\right)^{\log (z)} \log \left(t_{i}\right) \log \left(t_{j}\right), \\
\frac{\Theta^{2} F(\tilde{z})}{2}= & \frac{1}{2} \sum_{i=1}^{n}\left(\frac{\partial^{2} f}{\partial x_{i}^{2}}\left(\xi_{1}, \xi_{2}, \ldots, \xi_{n}\right) \xi_{i}^{2}+\frac{\partial f}{\partial x_{i}}\left(\xi_{1}, \xi_{2}, \ldots, \xi_{n}\right) \xi_{i}\right) \log ^{2}\left(t_{i}\right) \\
& +\frac{1}{2} \sum_{i, j=1, i \neq j}^{n} \frac{\partial^{2} f}{\partial x_{i} \partial x_{j}}\left(\xi_{1}, \xi_{2}, \ldots, \xi_{n}\right) \xi_{i} \xi_{j} \log \left(t_{i}\right) \log \left(t_{j}\right)
\end{aligned}
$$


with $\left(\xi_{1}, \xi_{2}, \ldots, \xi_{n}\right)=\left(t_{1}^{\log (\tilde{z})} x_{1}, \ldots, t_{n}^{\log (\tilde{z})} x_{n}\right) \in L_{t_{1}, \ldots, t_{n}}$.

Now, by using the definition of the partial Mellin derivative, we have

$$
\frac{\partial^{2} f}{\partial x_{i}^{2}}\left(\xi_{1}, \xi_{2}, \ldots, \xi_{n}\right) \xi_{i}^{2}=\left[\Theta_{x_{i}}^{2} f\left(\xi_{1}, \xi_{2}, \ldots, \xi_{n}\right)-\Theta_{x_{i}} f\left(\xi_{1}, \xi_{2}, \ldots, \xi_{n}\right)\right]
$$

Hence, we obtain

$$
\frac{\Theta^{2} F(\tilde{z})}{2}=\frac{1}{2}\left(\Theta_{x_{1}} \log \left(t_{1}\right)+\ldots+\Theta_{x_{n}} \log \left(t_{n}\right)\right)^{2} f\left(\xi_{1}, \xi_{2}, \ldots, \xi_{n}\right)
$$

which completes the proof.

By Proposition 1, we can derive the Mellin-Taylor formula with the Peano remainder as follows.

Proposition 2. Let the assumptions of Proposition 1 are satisfied. Then,

$$
\lim _{\mathbf{t} \rightarrow \mathbf{1}} \frac{\left(\Theta_{x_{1}} \log \left(t_{1}\right)+\ldots+\Theta_{x_{n}} \log \left(t_{n}\right)\right)^{m} f(\boldsymbol{\xi})-\left(\Theta_{x_{1}} \log \left(t_{1}\right)+\ldots+\Theta_{x_{n}} \log \left(t_{n}\right)\right)^{m} f(\mathbf{x})}{\left(\log ^{2}\left(t_{1}\right)+\ldots+\log ^{2}\left(t_{n}\right)\right)^{m / 2}}=0,
$$

where $\xi=\left(\xi_{1}, \ldots, \xi_{n}\right)$.

Proof. We prove for $m=2$, with a similar method in the previous proof (it can be applied for general case). Let

$$
I:=\left|\left(\Theta_{x_{1}} \log \left(t_{1}\right)+\ldots+\Theta_{x_{n}} \log \left(t_{n}\right)\right)^{2} f(\boldsymbol{\xi})-\left(\Theta_{x_{1}} \log \left(t_{1}\right)+\ldots+\Theta_{x_{n}} \log \left(t_{n}\right)\right)^{2} f(\mathbf{x})\right|,
$$

then we have

$$
\frac{I}{\log ^{2}\left(t_{1}\right)+\ldots+\log ^{2}\left(t_{n}\right)} \leq \sum_{i=1}^{n}\left|\Theta_{x_{i}}^{2} f(\boldsymbol{\xi})-\Theta_{x_{i}}^{2} f(\mathbf{x})\right|+\sum_{i=1, i \neq j}^{n-1}\left|\Theta_{x_{i}}\left(\Theta_{x_{j}} f\right)(\boldsymbol{\xi})-\Theta_{x_{i}}\left(\Theta_{x_{j}} f\right)(\mathbf{x})\right| .
$$

Considering $\xi \in L_{t_{1}, t_{2}, \ldots, t_{n}}$ the assertion follows from the assumption $f \in C^{(2)}\left(\mathbb{R}_{+}^{n}\right)$.

Now by Proposition 2, it is obvious to write the local form of the Mellin-Taylor formula as

$$
f(\mathbf{t x})=f(\mathbf{x})+\sum_{r=1}^{m} \frac{1}{r !}\left(\Theta_{x_{1}} \log \left(t_{1}\right)+\ldots+\Theta_{x_{n}} \log \left(t_{n}\right)\right)^{r} f(\mathbf{x})+R_{m}(\mathbf{t})
$$

with the Peano remainder

$$
R_{m}(\mathbf{t})=R_{m}\left(t_{1}, \ldots, t_{n}\right)=H\left(t_{1}, \ldots, t_{n}\right)\left(\log ^{2}\left(t_{1}\right)+\ldots+\log ^{2}\left(t_{n}\right)\right)^{m / 2},
$$

where $H\left(t_{1}, \ldots, t_{n}\right)$ is a bounded function such that

$$
\lim _{\left(t_{1}, \ldots, t_{n}\right) \rightarrow(1, \ldots, 1)} H\left(t_{1}, \ldots, t_{n}\right)=0
$$

Remark 3. Note that setting $\mathbf{t}=\left(t_{1}, \ldots, t_{n}\right), \mathbf{x}=\left(x_{1}, \ldots, x_{n}\right)$, it is easy to see that

$$
R_{m}(\mathbf{t})=H(\mathbf{t})\|\log (\mathbf{t} \mathbf{x})-\log (\mathbf{x})\|^{m} .
$$


Let $\mathbf{h}=\left(h_{1}, \ldots, h_{n}\right) \in \mathbb{N}_{0}^{n}$ and $v=\lfloor\mathbf{h}\rfloor=h_{1}+\ldots+h_{n}$. For $\mathbf{x} \in \mathbb{R}_{+}^{n}$, we define the moments of order $\mathbf{h}$ of $\varphi \in \Phi$ as

$$
\begin{aligned}
m_{\mathbf{h}}^{v}(\varphi, \mathbf{x}): & =\sum_{\mathbf{k} \in \mathbb{Z}^{n}} \varphi\left(e^{-\mathbf{k}} \mathbf{x}\right) \log ^{\mathbf{h}}\left(e^{\mathbf{k}} \mathbf{x}^{-1}\right)=\sum_{\mathbf{k} \in \mathbb{Z}^{n}} \varphi\left(e^{-\mathbf{k}} \mathbf{x}\right)\left\langle(\mathbf{k}-\log (\mathbf{x}))^{\mathbf{h}}\right\rangle \\
& =\sum_{\left(k_{1}, k_{2}, \ldots, k_{n}\right) \in \mathbb{Z}^{n}} \varphi\left(e^{-k_{1}} x_{1}, \ldots, e^{-k_{n}} x_{n}\right)\left(k_{1}-\log \left(x_{1}\right)\right)^{h_{1}} \ldots\left(k_{n}-\log \left(x_{n}\right)\right)^{h_{n}} .
\end{aligned}
$$

The absolute moments of order $\alpha>0$ of $\varphi \in \Phi$ is defined as

$$
M_{\alpha}(\varphi, \mathbf{x}):=\sum_{\mathbf{k} \in \mathbb{Z}^{n}}\left|\varphi\left(e^{-\mathbf{k}} \mathbf{x}\right)\right|\|\mathbf{k}-\log (\mathbf{x})\|^{\alpha} .
$$

Finally, we set $M_{\alpha}(\varphi):=\sup _{\mathbf{x} \in \mathbb{R}_{+}^{n}} M_{\alpha}(\varphi, \mathbf{x})$.

Now, we obtain estimations of the order of approximation under some local regularity assumptions on the function $f$. In order to do that, we need the following assumptions on the kernel function, i.e. there exists $l \in \mathbb{N}$ such that $\mathbf{h} \in \mathbb{N}_{0}^{n},\lfloor\mathbf{h}\rfloor \leq l$

$(\varphi .4) m_{\mathbf{h}}^{\lfloor\mathbf{h}\rfloor}(\varphi, \mathbf{x}):=m_{\mathbf{h}}^{\lfloor\mathbf{h}\rfloor}(\varphi)$ is independent of $\mathbf{x} ;$

$(\varphi .5) M_{l}(\varphi)<+\infty$ and

$$
\lim _{r \rightarrow+\infty} \sum_{\|\mathbf{k}-\log (\mathbf{x})\|>r}\left|\varphi\left(e^{-\mathbf{k}} \mathbf{x}\right)\right|\|\mathbf{k}-\log (\mathbf{x})\|^{l}=0
$$

uniformly with respect to $\mathbf{x}$.

We denote by $\Phi_{l}$ the set of functions $\varphi$ satisfying assumptions $(\varphi .1),(\varphi .4),(\varphi .5)$.

Then for $l=m$, we have the following Voronovskaja type result.

Theorem 4. Let $f: \mathbb{R}_{+}^{n} \rightarrow \mathbb{R}$ be a function such that $f \in C^{(m)}\left(\mathbb{R}_{+}^{n}\right)$ locally at the point $\mathbf{x}=$ $\left(x_{1}, \ldots, x_{n}\right) \in \mathbb{R}_{+}^{n}$ and $\varphi \in \Phi_{m}$. Then

$$
\left(\mathrm{E}_{\mathbf{w}}^{\varphi} f\right)(\mathbf{x})-f(\mathbf{x})=\sum_{r=1}^{m} \sum_{\lfloor\mathbf{h}\rfloor=r} \frac{1}{\mathbf{h} !} \Theta_{x_{1}^{h_{1}} x_{2}^{h_{2} \ldots} \ldots x_{n}^{h_{n}}}^{r} f(\mathbf{x})\left[\prod_{i=1}^{n} w_{i}^{-h_{i}}\right] m_{\mathbf{h}}^{r}(\varphi)+o\left(\frac{1}{\bar{w}^{m}}\right)
$$

as $\bar{w} \rightarrow+\infty$.

Proof. Since $f \in C^{(m)}\left(\mathbb{R}_{+}^{n}\right)$, by using Mellin-Taylor formula, we can write

$$
\begin{aligned}
\left(\mathrm{E}_{\mathbf{w}}^{\varphi} f\right)(\mathbf{x})= & \sum_{\mathbf{k} \in \mathbb{Z}^{n}} \varphi\left(e^{-\mathbf{k} \mathbf{x}^{\mathbf{w}}}\right)\left(f(\mathbf{x})+\sum_{r=1}^{m} \frac{1}{r !}\left[\Theta_{x_{1}} \log \left(\frac{e^{k_{1} / w_{1}}}{x_{1}}\right)+\ldots+\Theta_{x_{n}} \log \left(\frac{e^{k_{n} / w_{n}}}{x_{n}}\right)\right]^{r} f(\mathbf{x})\right. \\
& \left.+H\left(\frac{e^{k_{1} / w_{1}}}{x_{1}}, \ldots, \frac{e^{k_{n} / w_{n}}}{x_{n}}\right)\left\|\log \left(e^{\mathbf{k} / \mathbf{w}}\right)-\log (\mathbf{x})\right\|^{m}\right) .
\end{aligned}
$$

Now, by assumption $(\varphi .1)$ and using (3) we have

$$
\begin{aligned}
& \left(\mathrm{E}_{\mathbf{w}}^{\varphi} f\right)(\mathbf{x})-f(\mathbf{x})=\sum_{r=1}^{m} \frac{1}{r !} \sum_{\lfloor\mathbf{h}\rfloor=r} \frac{r !}{\mathbf{h} !} \Theta_{x_{1}}^{h_{1}}\left(\ldots\left(\Theta_{x_{n}}^{h_{n}} f\right)\right)(\mathbf{x}) \\
& \times \sum_{\mathbf{k} \in \mathbb{Z}^{n}}\left(\frac{k_{1}}{w_{1}}-\log \left(x_{1}\right)\right)^{h_{1}} \cdots\left(\frac{k_{n}}{w_{n}}-\log \left(x_{n}\right)\right)^{h_{n}} \varphi\left(e^{-\mathbf{k}} \mathbf{x}^{\mathbf{w}}\right)
\end{aligned}
$$

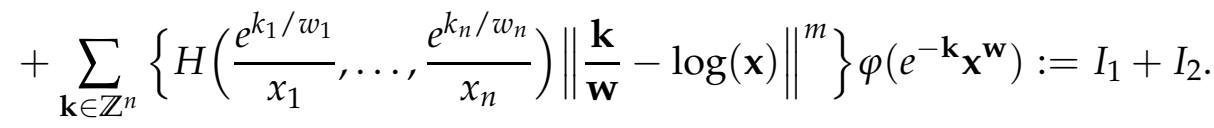


Hence, $I_{1}$ can be written in the form

$$
I_{1}=\sum_{r=1}^{m} \sum_{\lfloor\mathbf{h}\rfloor=r} \frac{1}{\mathbf{h} !} \Theta_{x_{1}}^{h_{1}}\left(\ldots\left(\Theta_{x_{n}}^{h_{n}} f\right)\right)(\mathbf{x})\left[\prod_{i=1}^{n} w_{i}^{-h_{i}}\right] m_{\mathbf{h}}^{r}(\varphi)
$$

Now, let us estimate $I_{2}$. For a given $\varepsilon>0$, there exists $\delta>0$ such that

$$
\left|H\left(\frac{e^{k_{1} / w_{1}}}{x_{1}}, \ldots, \frac{e^{k_{n} / w_{n}}}{x_{n}}\right)\right|<\varepsilon
$$

whenever $\left\|\log \left(e^{\mathbf{k} / \mathbf{w}}\right)-\log (\mathbf{x})\right\|=\left\|\frac{\mathbf{k}}{\mathbf{w}}-\log (\mathbf{x})\right\| \leq \delta$. Taking into account that

$$
\left\|\frac{\mathbf{k}}{\mathbf{w}}-\log (\mathbf{x})\right\| \leq \frac{\left\|\mathbf{k}-\log \left(\mathbf{x}^{\mathbf{w}}\right)\right\|}{\bar{w}},
$$

we obtain

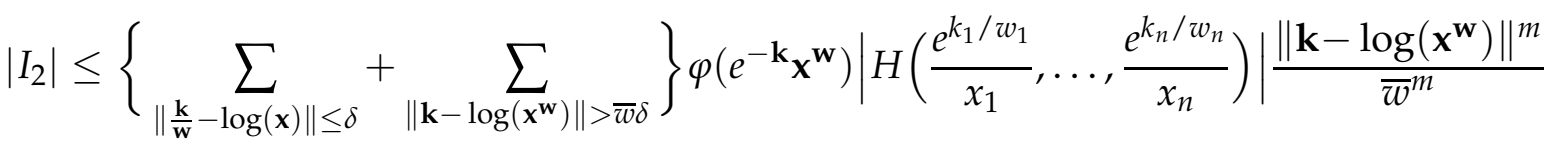

$$
\begin{aligned}
& :=I_{2,1}+I_{2,2} \text {. }
\end{aligned}
$$

By using the definition of $M_{m}(\varphi)$, we easily conclude that $\bar{w}^{m} I_{2,1}<\varepsilon M_{m}(\varphi)$. Finally, by $(\varphi .5)$ and by the boundedness of $H$, we get $\bar{w}^{m} I_{2,2} \leq\|H\|_{\infty} \varepsilon$ and this completes the proof as well.

\section{References}

[1] Acar T., Kursun S., Turgay M. Multidimensional Kantorovich modifications of exponential sampling series. Quaest. Math. 2021 (accepted).

[2] Bardaro C., Bevignani G., Mantellini I., Seracini M. Bivariate generalized exponential sampling series and applications to seismic waves. Constr. Math. Anal. 2019, 2 (4), 153-167. doi:10.33205/cma.594066

[3] Bardaro C., Butzer P.L., Mantellini I., Schmeisser G. On the Paley-Wiener theorem in the Mellin transform setting. J. Approx. Theory 2016, 207, 60-75. doi:10.1016/j.jat.2016.02.010

[4] Bardaro C., Butzer P.L., Mantellini I., Schmeisser G. A fresh approach to the Paley-Wiener theorem for Mellin transforms and the Mellin-Hardy spaces. Math. Nachr. 2017, 290 (17-18), 2759-2774. doi:10.1002/mana.201700043

[5] Bardaro C., Butzer P.L., Mantellini I. The exponential sampling theorem of signal analysis and the reproducing kernel formula in the Mellin transform setting. Sampl. Theory Signal Image Process. 2014, 13 (1), $35-66$. doi:10.1007/BF03549572

[6] Bardaro C., Butzer P.L., Mantellini I. The Mellin-Parseval formula and its interconnections with the exponential sampling theorem of optical physics. Integral Transforms Spec. Funct. 2016, 27 (1), 17-29. doi: 10.1080/10652469.2015.1087401

[7] Bardaro C., Faina L., Mantellini I. Quantitative Voronovskaja formulae for generalized Durrmeyer sampling type series. Math. Nachr. 2016, 289 (14-15), 1702-1720. doi: 10.1002/mana.201500225

[8] Bardaro C., Faina L., Mantellini I. A generalization of the exponential sampling series and its approximation properties. Math. Slovaca 2017, 67 (6), 1481-1496. doi:10.1515/ms-2017-0064

[9] Bardaro C., Mantellini I. A note on the Voronovskaja theorem for Mellin-Fejer convolution operators. Appl. Math. Lett. 2011, 24, 2064-2067. doi:10.1016/j.aml.2011.05.043 
[10] Bardaro C., Mantellini I. On Mellin convolution operators: a direct approach to the asymptotic formulae. Integral Transforms Spec. Funct. 2014, 25 (3), 182-195. doi:10.1080/10652469.2013.838755

[11] Bardaro C., Mantellini I. On a Durrmeyer-type modification of the exponential sampling series. Rend. Circ. Mat. Palermo, II. Ser. 2021, 70 (3), 1289-1304. doi:10.1007/s12215-020-00559-6

[12] Bardaro C., Mantellini I., Schmeisser G. Exponential sampling series: convergence in Mellin-Lebesgue spaces. Results Math. 2019, 74 (3), 119. doi:10.1007/s00025-019-1044-5

[13] Bardaro C., Vinti G. A general approach to the convergence theorems of generalized sampling series. Appl. Anal. 1997, 64, 203-217. doi:10.1080/00036819708840531

[14] Bertero M., Pike E.R. Exponential-sampling method for Laplace and other dilationally invariant transforms, II. Examples in photon correlation spectroscopy and Fraunhofer diffraction. Inverse Problems 1991, 7 (1), $21-41$.

[15] Butzer P.L., Fischer A., Stens R.L. Generalized sampling approximation of multivariate signals; general theory. Atti Sem. Mat. Fis. Univ. Modena 1993, 41 (1), 17-37.

[16] Butzer P.L., Fischer A., Stens R.L. Generalized sampling approximation of multivariate signals; theory and some applications. Note Mat. 1990, 10 (1), 173-191. doi:10.1285/i15900932v10supn1p173

[17] Butzer P.L., Jansche S. A direct approach to the Mellin transform. J. Fourier Anal. Appl. 1997, 3 (4), $325-375$. doi:10.1007/BF02649101

[18] Butzer P.L., Nessel R.J. Fourier Analysis and Approximation I. Academic press, New York-London, 1971.

[19] Butzer P.L., Splettstösser W., Stens R.L. The sampling theorem and linear prediction in signal analysis. Jahresber. Deutsch. Math.-Ver. 1988, 90, 1-70.

[20] Butzer P.L., Ries S., Stens R.L. Approximation of continuous and discontinuous functions by generalized sampling series. J. Approx. Theory 1987, 50 (1), 25-39. doi:10.1016/0021-9045(87)90063-3

[21] Gori F. Sampling in optics. Advanced topics in Shannon sampling and interpolation theory. Springer, New York, 1993, 37-83.

[22] Higgins J.R. Sampling theory in Fourier and signal analysis: Foundations. Oxford Univ. Press, Oxford, 1996.

[23] Mamedov R.G. The Mellin transform and approximation theory. Elm, Baku, 1991. (in Russian)

[24] Ries S., Stens R.L. Approximation by generalized sampling series. In: Proc. of the Intern. Conf. on Constructive Theory of Functions, Varna, Bulgaria, 1984, Bulgarian Academy of Science, Sofia, 1984, 746-756.

[25] Zayed A.I. Advances in Shannon's Sampling Theory. CRC Press, Boca Raton, 1993.

Курсун С., Тургай М., Алагоз О., Акар Т. Апроксимачійні властивості багатовимірних експоненціальних вибіркових рядів // Карпатські матем. публ. - 2021. - Т.13, №3. - С. 666-675.

У цій роботі узагальнено сім'ю експоненціальних вибіркових рядів для функцій $n$ змінних та досліджено їх точкову і рівномірну збіжність, а також швидкість збіжності для функцій, що належать до простору log-рівномірно неперервних функцій. Крім того, сформульовано та доведено узагальнене розвинення Мелліна-Тейлора багатовимірних функцій і за допомогою теореми типу Вороновської встановлено точкову асимптотичну поведінку ряду.

Ключові слова і фрази: багатовимірний експоненщіальний вибірковий ряд, швидкість збіжності, багатовимірна формула Мелліна-Тейлора, теорема типу Вороновської. 